Polymer Journal, Vol. 39, No. 12, pp. 1373-1382 (2007)

(C) 2007 The Society of Polymer Science, Japan

\title{
A Monte Carlo Study of the Intrinsic Viscosity of Semiflexible Regular Three-Arm Star Polymers
}

\author{
Daichi IDA and Takenao YosHIZAKI ${ }^{\dagger}$
}

Department of Polymer Chemistry, Kyoto University, Katsura Kyoto 615-8510, Japan

(Received August 20, 2007; Accepted September 14, 2007; Published October 31, 2007)

\begin{abstract}
A Monte Carlo (MC) study is made of the intrinsic viscosity $[\eta]$ and also of the mean-square radius of gyration $\left\langle S^{2}\right\rangle$ for regular three-arm star freely rotating chains of bond angles $\theta=109^{\circ}, 165^{\circ}$, and $175^{\circ}$ and with the Lennard-Jones 6-12 potentials between beads having the parameter values corresponding to the $\Theta$ temperature, in the range of the total number $n$ of bonds in the chain from 60 to 300. Three kinds of approximate values of $[\eta]$ are calculated by the use of the Kirkwood-Riseman (KR) approximation, the Zimm rigid-body ensemble approximation which gives an upper bound $[\eta]^{(\mathrm{U})}$ to $[\eta]$, and by the Fixman method which gives a lower bound $[\eta]^{(\mathrm{L})}$, the KR value of $[\eta]$ being designated $[\eta]^{(\mathrm{KR})}$. On the basis of the three kinds of MC values of $[\eta]$ so obtained, the behavior of the ratio $g_{\eta}$ of $[\eta]$ for the star chain to that for the linear one, both having the same $n$, is examined as a function of the reduced contour length $\lambda L$ as defined as the total contour length $L$ of the corresponding Kratky-Porod (KP) wormlike chain divided by its stiffness parameter $\lambda^{-1}$, the values of $\lambda L$ having been determined from an analysis of the present and previous MC data for $\left\langle S^{2}\right\rangle$ on the basis of the KP chain. It is found that the KR value $g_{\eta}^{(\mathrm{KR})}$ of $g_{\eta}$ as defined by $[\eta]^{(\mathrm{KR})}(\mathrm{star}) /$ $[\eta]^{(\mathrm{KR})}\left(\right.$ linear) lies between the values of an upper bound $g_{\eta}^{(\mathrm{U})}$ and a lower one $g_{\eta}^{(\mathrm{L})}$, which are defined by $[\eta]^{(\mathrm{U})}($ star $) /[\eta]^{(\mathrm{L})}$ (linear) and $[\eta]^{(\mathrm{L})}($ star $) /[\eta]^{(\mathrm{U})}$ (linear), respectively, irrespective of the values of $\lambda L$. Further, the difference between the two bounds becomes very small for small $\lambda L$, indicating that $g_{\eta}^{(\mathrm{KR})}$ may give a good approximate value for semiflexible or stiff chains. [doi:10.1295/polymj.PJ2007121]

KEY WORDS Three-Arm Star Polymer / Intrinsic Viscosity / Monte Carlo Simulation / Freely Rotating Chain / Semiflexible Polymer / Wormlike Chain /
\end{abstract}

The mean-square radius of gyration $\left\langle S^{2}\right\rangle$ and/or the intrinsic viscosity $[\eta]$ as measures of the average chain dimension of polymers in dilute solution depend largely on their molecular structure, e.g., linear or branched. It has therefore been of great interest to investigate the difference in $\left\langle S^{2}\right\rangle$ or $[\eta]$ between linear and branched polymer chains, and many theoretical and experimental studies ${ }^{1-9}$ have already been made. However, almost all of those studies were of flexible polymers except for the theoretical one by Mansfield and Stockmayer, ${ }^{4}$ the Monte Carlo (MC) one by Zimm, ${ }^{5}$ and the experimental one by Goodson and Novak. ${ }^{9}$ Mansfield and Stockmayer calculated $\left\langle S^{2}\right\rangle$ of the Kratky-Porod (KP) wormlike ${ }^{10,11}$ star chain (without excluded volume) and examined the behavior of its ratio $g_{S}$ to $\left\langle S^{2}\right\rangle$ of the corresponding linear one having the same total length of the chain contour (molecular weight). Zimm evaluated $[\eta]$ of "wormlike" four- and six-arm stars by the use of his rigidbody ensemble approximation. ${ }^{12}$ Unfortunately, however, his calculation is rather limited, as mentioned by himself that it was a preliminary survey. Goodson and Novak synthesized three-arm star poly $(n$-hexyl isocyanate) by living titanium-catalized coordination polymerization, ${ }^{9}$ and then investigated the behavior of $\left\langle S^{2}\right\rangle,[\eta]$, and $g_{S}$ as functions of molecular weight.
In this paper, we make an extensive MC study of $[\eta]$ for semiflexible regular three-arm star polymers in the $\Theta$ state, which has not yet been made.

The regular three-arm star chain model used in this study is essentially the same as that used in previous MC studies ${ }^{13,14}$ of $\left\langle S^{2}\right\rangle$ and the second virial coefficient of linear chains with intra- and intermolecular excluded volume, i.e., the freely rotating chain 3,11 with a cutoff version of the Lennard-Jones (LJ) 6-12 potential $^{15}$ between beads. On the basis of sets of sample configurations generated by the use of the Metropolis method ${ }^{16}$ with the pivot algorithm ${ }^{17-19}$ for the regular three-arm star and linear chains, both having the same total chain length, the values of $[\eta]$ for the two kinds of chains and then the ratio $g_{\eta}$ of the former $[\eta]$ value to the latter may be evaluated.

The evaluation of $[\eta]$ requires some comments. As is well known, there is no method for the exact evaluation of $[\eta]$ since the hydrodynamic interaction between the segments composing polymer chains cannot be treated properly. We therefore evaluate $[\eta]$ in the three approximate ways: the Kirkwood-Riseman (KR) approximation, ${ }^{3,20}$ the Zimm rigid-body ensemble approximation, ${ }^{12}$ and the Fixman method. ${ }^{21,22} \mathrm{We}$ note that the Zimm method gives an upper bound to $[\eta]$ and the Fixman method gives a lower bound. On

${ }^{\dagger}$ To whom correspondence should be addressed (E-mail: yoshizaki@molsci.polym.kyoto-u.ac.jp). 


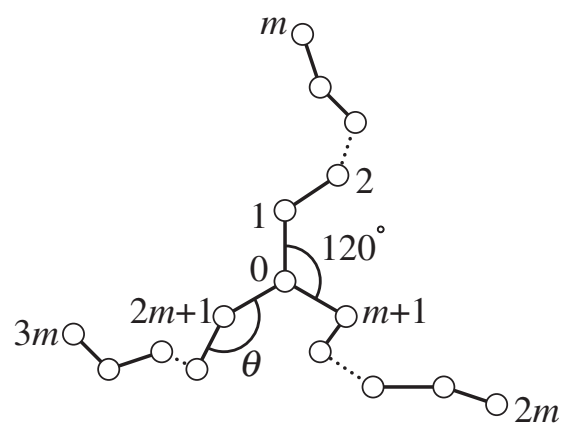

Figure 1. Illustration of the Monte Carlo model for the regular three-arm star chain.

the basis of the three kinds of MC values, we examine the behavior of $[\eta]$ and $g_{\eta}$ as functions of the total chain length and the chain stiffness.

\section{MODEL AND METHODS}

\section{Model}

The MC model used in this study is the regular three-arm star freely rotating chain, each arm composed of $m$ successive bonds of length unity, so that it is composed of $3 m(=n)$ bonds, in total, and $3 m+1$ beads whose centers are located at $3 m-3$ junctions of two successive bonds on the arms, at the three terminal ends, and at the branch point (center), as illustrated in Figure 1. The angle between each pair of the bonds connected to the center is fixed to be $120^{\circ}$, so that those bonds are on the same plane. For convenience, the three arms are designated the first, second, and third ones and the $m$ beads on the $i$ th arm $(i=1$, $2,3)$ are numbered $(i-1) m+1,(i-1) m+2, \cdots$, im from the center to the end, with the center bead numbered 0 . The $i$ th bond vector $\mathbf{l}_{i}(1 \leq i \leq 3 m ; i \neq m+1$, $2 m+1)$ connects the centers of the $(i-1)$ th and $i$ th beads with its direction from the $(i-1)$ th to $i$ th bead, and $\mathbf{l}_{m+1}$ and $\mathbf{l}_{2 m+1}$ are from the 0th to the $(m+1)$ th and $(2 m+1)$ th beads, respectively. All the $3 m-3$ bond angles $\theta$ (not supplement) except for those around the center are fixed, so that the configuration of the entire chain may be specified by the set of $3 m-3$ rotation angles $\left\{\phi_{3 m-3}\right\}=\left(\phi_{1}, \cdots, \phi_{m-1}\right.$, $\left.\phi_{m+1}, \cdots, \phi_{2 m-1}, \phi_{2 m+1}, \cdots, \phi_{3 m-1}\right)$ apart from its position and orientation in an external Cartesian coordinate system, where $\phi_{i}$ is the internal rotation angle around $\mathbf{l}_{i}$.

The linear chain model, the counterpart of the above star one, is the same as that used in refs 13 and 14 , i.e., the freely rotating chain composed of $n$ bonds of length unity and $n+1$ beads, whose centers are located at the $n-1$ junctions of two successive bonds and at the two terminal ends. We set $n$ equal to $3 m$. The beads are numbered $0,1,2, \cdots, n$ from one end to the other, and $\mathbf{l}_{i}(1 \leq i \leq n)$ connects the centers of the $(i-1)$ th and $i$ th beads with its direction from the $(i-1)$ th to the $i$ th bead. All the $n-1$ bond angles are fixed at $\theta$, so that the configuration of the linear chain may be specified by the set of $n-2$ internal rotation angles $\left\{\phi_{n-2}\right\}=\left(\phi_{2}, \phi_{3}, \cdots, \phi_{n-1}\right)$ apart from its position and orientation in the external Cartesian coordinate system.

The total potential energy $U$ of the regular threearm star chain as a function of $\left\{\phi_{3 m-3}\right\}$ may be given by

$$
\begin{aligned}
U\left(\left\{\phi_{3 m-3}\right\}\right)= & \sum_{i=0}^{1} \sum_{j=i+1}^{2} \sum_{k, l=1}^{m} h(k+l-4) u\left(R_{(i m+k)(j m+l)}\right) \\
& +\sum_{i=0}^{2} \sum_{j=1}^{m-4} \sum_{k=j+4}^{m} u\left(R_{(i m+j)(i m+k)}\right) \\
& +\sum_{i=0}^{2} \sum_{j=4}^{m} u\left(R_{0(i m+j)}\right) \\
& \text { (regular three-arm star) }
\end{aligned}
$$

and that of the linear chain as a function of $\left\{\phi_{n-2}\right\}$ by

$$
U\left(\left\{\phi_{n-2}\right\}\right)=\sum_{i=0}^{n-4} \sum_{j=i+4}^{n} u\left(R_{i j}\right) \quad \text { (linear) }
$$

where $h(x)$ is a unit step function such that $h(x)=1$ for $x \geq 0$ and $h(x)=0$ for $x<0$ and $R_{i j}$ is the distance between the centers of the $i$ th and $j$ th beads. We note that in eqs 1 and 2 the interactions between the thirdneighbor beads along the chain have been neglected, since they seem to make the chain locally take the cis conformation to excess. We adopt as the pair potential $u(R)$ (of mean force) the cutoff version of the LJ 6-12 potential given by

$$
\begin{aligned}
u(R) & =\infty & & \text { for } 0 \leq R<c \sigma \\
& =u^{\mathrm{LJ}}(R) & & \text { for } c \sigma \leq R<3 \sigma \\
& =0 & & \text { for } 3 \sigma \leq R
\end{aligned}
$$

where $u^{\mathrm{LJ}}(R)$ is the $\mathrm{LJ}$ potential ${ }^{15}$ given by

$$
u^{\mathrm{LJ}}(R)=4 \epsilon\left[\left(\frac{\sigma}{R}\right)^{12}-\left(\frac{\sigma}{R}\right)^{6}\right]
$$

with $\sigma$ and $\epsilon$ the collision diameter and the depth of the potential well at the minimum of $u^{\mathrm{LJ}}(R)$, respectively. We note that $u(R)$ given by eqs 3 is the LJ potential cut off at the upper bound $3 \sigma$. The lower bound $c \sigma$ in eqs 3 has been introduced for numerical convenience; the factor $c$ is properly chosen so that the Boltzmann factor $e^{-u^{\mathrm{LJ}} / k_{\mathrm{B}} T}$ may be regarded as numerically vanishing compared to unity, where $k_{\mathrm{B}}$ is the Boltzmann constant and $T$ is the absolute temperature. In practice, in double-precision computation, we put 


$$
c=\left[2 /\left(1+\sqrt{1+36 T^{*}}\right)\right]^{1 / 6}
$$

so that $e^{-u^{\mathrm{LJ}} / k_{\mathrm{B}} T} \lesssim 2 \times 10^{-16}$ for $0 \leq R<c \sigma$, where $T^{*}$ is the reduced temperature defined by $T^{*}=$ $k_{\mathrm{B}} T / \epsilon$. Further, we put $\sigma=1$ for simplicity, as previously ${ }^{13,14}$ done.

\section{Sampling}

The MC simulation algorithm used in this study is the same as that used in ref 13 and 14 and is essentially the same as the Stellman-Gans ${ }^{18}$ version of the pivot algorithm ${ }^{17,19}$ for a sequential generation of chain configurations. A brief description is given of the algorithm for the regular three-arm star chain, which is in principle the same as that for the linear chain described previously. ${ }^{13}$

First, we generate an initial configuration $\left\{\mathbf{I}_{3 m}\right\}=$ $\left(\mathbf{l}_{1}, \mathbf{l}_{2}, \cdots, \mathbf{l}_{3 m}\right)$ by trial and error. A trial set of the rotation angles $\left\{\phi_{3 m-3}\right\}$ are randomly generated in the interval $[-\pi, \pi]$. The branch point of the star chain is located at the origin of the external Cartesian coordinate system and the first bond vector $\mathbf{l}_{i m+1}(i=0$, $1,2)$ on the $(i+1)$ th arm is laid in the $x z$ plane such that $\mathbf{l}_{1}^{T}=(0,0,1), \mathbf{l}_{m+1}^{T}=\left(\frac{\sqrt{3}}{2}, 0,-\frac{1}{2}\right)$, and $\mathbf{l}_{2 m+1}^{T}=$ $\left(-\frac{\sqrt{3}}{2}, 0,-\frac{1}{2}\right)$, with the superscript $T$ indicating the transpose. The succeeding bond vector $\mathbf{l}_{i m+j}(j=2$, $3, \cdots, m)$ on the $(i+1)$ th arm may be given by

$$
\begin{gathered}
\mathbf{l}_{i m+j}=\mathbf{C}_{i} \cdot \mathbf{A}\left(\theta, \phi_{i m+1}\right) \cdot \mathbf{A}\left(\theta, \phi_{i m+2}\right) \cdots \\
\mathbf{A}\left(\theta, \phi_{i m+j-1}\right) \cdot(0,0,1)^{T}
\end{gathered}
$$

where $\mathbf{C}_{i}(i=0,1,2)$ is the orthogonal transformation matrix given by

$$
\mathbf{C}_{i}=\left(\begin{array}{ccc}
-1 / 2 & 0 & \sqrt{3} / 2 \\
0 & 1 & 0 \\
-\sqrt{3} / 2 & 0 & -1 / 2
\end{array}\right)^{i}
$$

and $\mathbf{A}\left(\theta, \phi_{i-1}\right)$ is the orthogonal transformation matrix from the $i$ th to the $(i-1)$ th localized Cartesian coordinate system ${ }^{11}$ given by

$$
\mathbf{A}(\theta, \phi)=\left(\begin{array}{ccc}
\cos \theta \cos \phi & \sin \phi & -\sin \theta \cos \phi \\
\cos \theta \sin \phi & -\cos \phi & -\sin \theta \sin \phi \\
-\sin \theta & 0 & -\cos \theta
\end{array}\right) \text { (8) }
$$

The $i$ th localized system associated with $\mathbf{l}_{i}(2 \leq$ $i \leq 3 m ; i \neq m+1,2 m+1)$ is defined as follows. The $z_{i}$ axis is taken along $\mathbf{l}_{i}$, the $x_{i}$ axis is in the plane of $\mathbf{l}_{i-1}$ and $\mathbf{l}_{i}$ with its direction chosen at an acute angle with $\mathbf{l}_{i-1}$, and the $y_{i}$ axis completes the right-handed system, so that $\phi_{i}=0$ in the trans conformation. Since the branch point is located at the origin, the vector position $\mathbf{r}_{i m+j}(i=0,1,2 ; j=1,2, \cdots, m)$ of the center of the $j$ th bead on the $(i+1)$ th arm is given by

$$
\mathbf{r}_{i m+j}=\sum_{k=1}^{j} \mathbf{l}_{i m+k}
$$

If all the distances between the centers of beads are greater than or equal to $c \sigma$, the above-given trial configuration is adopted as the initial configuration. If not, this trial is repeated until an initial satisfactory configuration is obtained.

Next the initial configuration $\left\{\mathbf{l}_{3 m}\right\}$ so obtained is sequentially changed by the pivot algorithm. A trial configuration is generated by rotating the chain with a given present configuration by an angle $\Delta \phi$ randomly chosen in the interval $[-\pi, \pi]$ around a bond randomly chosen from the $3 m-3$ bonds $\mathbf{l}_{i}(1 \leq i \leq$ $3 m-1 ; i \neq m, 2 m)$. If the $p$ th bond is chosen, the set of rotation angles $\left\{\phi_{3 m-3}^{\prime}\right\}$ in the trial configuration are given by

$$
\phi_{i}^{\prime}=\phi_{i}+\delta_{i p} \Delta \phi \quad(1 \leq i \leq 3 m-1 ; i \neq m, 2 m)
$$

with $\delta_{i p}$ the Kronecker delta. Practically, the trial configuration $\left\{\mathbf{I}_{3 m}^{\prime}\right\}$ is generated from $\left\{\phi_{3 m-3}^{\prime}\right\}$ by the use of the numerical procedure described previously. ${ }^{13}$ Then, the adoption of the trial configuration so generated as the next one is determined by the Metropolis method of importance sampling ${ }^{16}$ on the basis of the total potential energy given by eq 1 for the trial and present configurations, as described previously. ${ }^{13}$

By the use of the model and algorithm described above for the regular three-arm star chain and previously for the linear chain, ${ }^{13}$ we sample one configuration at every $M_{\text {nom }}$ (nominal) pivot steps, and $N$ configurations in total, for both chains. Then, the ensemble average $\langle A\rangle$ of a variable $A$ may be evaluated on the basis of the $N$ sample configurations so generated. If $A$ is independent of the orientation of the chain like the squared radius of gyration $S^{2}$, it is a function only of $\left\{\phi_{\alpha}\right\}$ with $\alpha$ being equal to $3 m-3$ for the star chain and $n-2$ for the linear one, so that $\langle A\rangle$ may be evaluated from

$$
\langle A\rangle=N^{-1} \sum_{\left\{\phi_{\alpha}\right\}} A\left(\left\{\phi_{\alpha}\right\}\right)
$$

where the sum is taken over the $N$ sample configurations. If $A$ is dependent on the orientation, on the other hand, the above average must be evaluated after randomizing the orientation for each sample configuration. Note that $N \times M_{\text {nom }}$ pivot steps are required to carry out a MC run in both cases. All the MC runs have been carried out at the reduced temperature $T^{*}=$ $3.72(\Theta)$ at which $\left\langle S^{2}\right\rangle / n$ for the linear chains with $\sigma=1$ and $\theta=109^{\circ}$ becomes a constant independent of $n$ for very large $n .^{13}$

All numerical work has been done by the use of a personal computer with an AMD Athlon XP 2600+ CPU. A source program coded in $\mathrm{C}$ has been compiled by the GNU C compiler version 2.95 .3 with real variables of double precision. For a generation of pseudorandom numbers, we have used the subrou- 
tine package MT19937 supplied by Matsumoto and Nishimura ${ }^{23}$ instead of the subroutine RAND included in the standard C library.

\section{Mean-square Radius of Gyration}

The mean-square radius of gyration $\left\langle S^{2}\right\rangle$ has been evaluated from eq 11, where $S^{2}$ for each sample chain has been calculated from

$$
S^{2}=\frac{1}{n+1} \sum_{i=0}^{n} S_{i}^{2}
$$

where $S_{i}$ is the magnitude of the vector distance $\mathbf{S}_{i}$ of the center of the $i$ th bead from the center of mass of the chain, i.e.,

$$
\mathbf{S}_{i}=\mathbf{r}_{i}-\mathbf{r}_{\mathrm{c} . \mathrm{m} .}
$$

with $\mathbf{r}_{\mathrm{c} . \mathrm{m} \text {. }}$ the vector position of the center of mass of the sample chain given by

$$
\mathbf{r}_{\text {c.m. }}=\frac{1}{n+1} \sum_{i=0}^{n} \mathbf{r}_{i}
$$

\section{Intrinsic Viscosity}

The intrinsic viscosity $[\eta]$ of the bead model without the contribution of each single-bead (i.e., without consideration of a distribution of frictional force on the surface of each bead) may be written in terms of the (total) frictional force $\mathbf{F}_{i}^{T}=\left(F_{i x}, F_{i y}, F_{i z}\right)$ exerted by the $i$ th bead on a surrounding solvent and $\mathbf{S}_{i}^{T}=$ $\left(S_{i x}, S_{i y}, S_{i z}\right)$ defined by eq 13 as follows, ${ }^{3}$

$$
[\eta]=-\frac{N_{\mathrm{A}}}{2 M \eta_{0} g} \sum_{i=0}^{n}\left\langle F_{i y} S_{i x}+F_{i x} S_{i y}\right\rangle
$$

where $N_{\mathrm{A}}$ is the Avogadro constant, $M$ is the molecular weight, $\eta_{0}$ is the viscosity coefficient of the solvent, and $g$ is the velocity gradient of a simple shear flow field. The frictional force $\mathbf{F}_{i}$ satisfies the following hydrodynamic equations,

$$
\begin{array}{r}
\mathbf{F}_{i}=\zeta\left(\mathbf{u}_{i}-\mathbf{v}_{i}^{0}\right)-\zeta \sum_{\substack{j=0 \\
\neq i}}^{n} \mathbf{T}_{i j} \cdot \mathbf{F}_{j} \\
(i=0,1,2, \cdots, n)
\end{array}
$$

where $\zeta$ is the friction coefficient of the bead given by the Stokes relation $\zeta=3 \pi \eta_{0} d_{\mathrm{b}}$ with $d_{\mathrm{b}}$ the hydrodynamic diameter of the bead, $\mathbf{u}_{i}$ is the velocity of the $i$ th bead, $\mathbf{v}_{i}^{0}$ is the unperturbed velocity of the solvent at the center of the $i$ th bead, and $\mathbf{T}_{i j}$ is the Oseen tensor representing the hydrodynamic interaction between the $i$ th and $j$ th beads.

In the numerical evaluation of $[\eta]$, the linear simultaneous equations 16 are first solved for $\mathbf{F}_{i}$ for the polymer chain immersed in the solvent having the unperturbed flow field,

$$
\mathbf{v}_{i}^{0}=g \mathbf{e}_{x} \mathbf{e}_{y} \cdot \mathbf{r}_{i}
$$

in the external Cartesian coordinate system $\left(\mathbf{e}_{x}, \mathbf{e}_{y}, \mathbf{e}_{z}\right)$, and then the ensemble averages on the right-hand side of eq 15 are evaluated by the use of the values of $\mathbf{F}_{i}$ so obtained. Since $[\eta]$ cannot be evaluated exactly, as mentioned already, it has been approximately evaluated in the three ways: the KR approximation, ${ }^{3,20}$ the Zimm rigid-body ensemble approximation, ${ }^{12}$ and the Fixman method. ${ }^{21,22}$ In what follows, we consider the touched-bead model so that $d_{\mathrm{b}}=1$.

$K R$ Approximation. In the KR approximation, the polymer chain in the unperturbed flow filed given by eq 17 is assumed to move with the translational velocity equal to that of the unperturbed flow at its center of mass and to rotate around its center of mass with the angular velocity $-\frac{1}{2} g \mathbf{e}_{z}$, so that $\mathbf{u}_{i}$ in eq 16 may be given by

$$
\mathbf{u}_{i}=\frac{1}{2} g\left(\mathbf{e}_{x} \mathbf{e}_{y}-\mathbf{e}_{y} \mathbf{e}_{x}\right) \cdot \mathbf{S}_{i}
$$

Further, the Oseen tensor defined by

$$
\mathbf{T}_{i j}=\frac{1}{8 \pi \eta_{0} R_{i j}}\left(\mathbf{I}+\frac{\mathbf{R}_{i j} \mathbf{R}_{i j}}{R_{i j}{ }^{2}}\right)
$$

with $\mathbf{I}$ the unit tensor and $\mathbf{R}_{i j}=\mathbf{S}_{j}-\mathbf{S}_{i}$ is replaced by its ensemble average $\left\langle\mathbf{T}_{i j}\right\rangle$, which may be written in the form,

$$
\left\langle\mathbf{T}_{i j}\right\rangle=\frac{1}{6 \pi \eta_{0}}\left\langle{R_{i j}}^{-1}\right\rangle \mathbf{I}
$$

In practice, $[\eta]$ in the KR approximation, which we designate $[\eta]^{(\mathrm{KR})}$, has been evaluated numerically as follows: First evaluate the average $\left\langle R_{i j}{ }^{-1}\right\rangle$ by the use of the $N$ sample chains, then solve the linear simultaneous equations 16 with $\left\langle\mathbf{T}_{i j}\right\rangle$ so obtained in place of $\mathbf{T}_{i j}$ for each sample chain having a random orientation with respect to the external system, and finally calculate $[\eta]^{(\mathrm{KR})}$ from eq 15 . We note that this numerical recipe correctly gives $[\eta]^{(\mathrm{KR})}$, although it seems apparently somewhat different from the original one., ${ }^{3,20}$

Rigid-Body Ensemble Approximation. The rigidbody ensemble approximation has been proposed by Zimm $^{12}$ in order to evaluate $[\eta]$ by MC simulation, in which each sample chain having a rigid conformation immersed in the shear flow given by eq 17 is assumed to move with an unknown translational velocity $\mathbf{U}$ and to rotate around its center of mass with the angular velocity $-\frac{1}{2} g \mathbf{e}_{z}$ under the condition that the total frictional force $\mathbf{F}$ exerted by the chain on the solvent vanishes. Then $\mathbf{u}_{i}$ in eq 16 may be given by

$$
\mathbf{u}_{i}=\mathbf{U}+\frac{1}{2} g\left(\mathbf{e}_{x} \mathbf{e}_{y}-\mathbf{e}_{y} \mathbf{e}_{x}\right) \cdot \mathbf{S}_{i}
$$

In this approximation, $\mathbf{F}_{i}$ and also $\mathbf{U}$ are evaluated for each sample chain having a random orientation by solving the linear simultaneous equations 16 with $\mathbf{u}_{i}$ 
and $\mathbf{v}_{i}^{0}$ given by eqs 21 and 17, respectively, along with the condition,

$$
\mathbf{F}=\sum_{i=0}^{n} \mathbf{F}_{i}=\mathbf{0}
$$

Then $[\eta]$ may be calculated from eq 15 with $\mathbf{F}_{i}$ so evaluated.

In the practical evaluation of $[\eta]$, we have used the modified Oseen tensor ${ }^{24,25} \mathbf{T}_{\mathrm{m}, i j}$ defined by

$$
\mathbf{T}_{\mathrm{m}, i j}=\mathbf{T}_{i j}+\frac{1}{16 \pi \eta_{0} R_{i j}}\left(\frac{d_{\mathrm{b}}}{R_{i j}}\right)^{2}\left(\frac{1}{3} \mathbf{I}-\frac{\mathbf{R}_{i j} \mathbf{R}_{i j}}{R_{i j}^{2}}\right)
$$

in place of the (original) Oseen tensor $\mathbf{T}_{i j}$ in order to avoid possible numerical troubles, i.e., the Zwanzig singularities ${ }^{26,27}$ associated with the touched-bead model. We note that $\left\langle\mathbf{T}_{\mathrm{m}, i j}\right\rangle$ becomes identical with $\left\langle\mathbf{T}_{i j}\right\rangle$.

Since the rigid-body ensemble approximation gives an upper bound to $[\eta]$, as pointed out by Wilemski and Tanaka $^{28}$ and by Fixman, ${ }^{21}[\eta]$ obtained in this approximation is designated $[\eta]^{(\mathrm{U})}$.

Fixman Method. Fixman ${ }^{21}$ has proposed an MC method for evaluating a lower bound $[\eta]^{(\mathrm{L})}$ to $[\eta]$. On the basis of a formal expression for $[\eta]$ by the use of the formal solution of the diffusion equation for the polymer chain, he has derived the following inequality,

$$
\begin{aligned}
{[\eta] \geq } & \frac{N_{\mathrm{A}}}{M \eta_{0} g^{2} k_{\mathrm{B}} T}\left[2\left\langle\mathbf{V}^{T} \cdot \mathbf{D}_{\mathrm{a}}^{-1} \cdot \mathbf{V}\right\rangle\right. \\
& \left.-\left\langle\mathbf{V}^{T} \cdot \mathbf{D}_{\mathrm{a}}^{-1} \cdot \mathbf{D} \cdot \mathbf{D}_{\mathrm{a}}^{-1} \cdot \mathbf{V}\right\rangle\right]
\end{aligned}
$$

where $\mathbf{V}$ is the column matrix with the $i$ th element $\mathbf{V}_{i}$ given by

$$
\mathbf{V}_{i}=\frac{1}{2} g\left(\mathbf{e}_{x} \mathbf{e}_{y}+\mathbf{e}_{y} \mathbf{e}_{x}\right) \cdot \mathbf{S}_{i}
$$

$\mathbf{D}$ is the $3(n+1) \times 3(n+1)$ (true) diffusion matrix whose $i j$ element is the $3 \times 3$ matrix $\mathbf{D}_{i j}$ defined by

$$
\mathbf{D}_{i j}=k_{\mathrm{B}} T\left[\zeta^{-1} \delta_{i j} \mathbf{I}+\left(1-\delta_{i j}\right) \mathbf{T}_{\mathrm{m}, i j}\right]
$$

and $\mathbf{D}_{\mathrm{a}}$ is an approximate expression for $\mathbf{D}$, which is desired to be easier to handle. Both the two tensors $\mathbf{D}$ and $\mathbf{D}_{\mathrm{a}}$ are required to be positive definite, symmetric, and divergenceless, and the former given by eq 26 satisfies the requirements. We note that in eq 24 equality holds if $\mathbf{D}_{\mathrm{a}}=\mathbf{D}$.

For any given expression for $\mathbf{D}_{\mathrm{a}}$ satisfying the above requirements, the two kinds of averages on the right-hand side of eq 24 may be evaluated on the basis of the MC sample configurations. The remaining problem is to find a tractable expression for $\mathbf{D}_{\mathrm{a}}$, for which Fixman has proposed the following expression, ${ }^{22}$

$$
\mathbf{D}_{\mathrm{a}}^{-1}=\alpha\left(\mathbf{D}_{\mathrm{p}}^{-1}+\mathbf{D}_{\mathrm{p}}^{\prime-1}\right)
$$

In this expression, $\mathbf{D}_{\mathrm{p}}$ and $\mathbf{D}_{\mathrm{p}}^{\prime}$ the $3(n+1) \times 3(n+1)$ matrices whose $i j$ elements are the $3 \times 3$ matrices given by

$$
\begin{aligned}
& \mathbf{D}_{\mathrm{p}, i j}=k_{\mathrm{B}} T\left[\zeta^{-1} \delta_{i j} \mathbf{I}+\left(1-\delta_{i j}\right)\left\langle\mathbf{T}_{i j}\right\rangle\right] \\
& \mathbf{D}_{\mathrm{p}, i j}^{\prime}=k_{\mathrm{B}} T\left[(\beta \zeta)^{-1} \delta_{i j} \mathbf{I}+\left(1-\delta_{i j}\right)\left\langle\mathbf{T}_{i j}\right\rangle\right]
\end{aligned}
$$

respectively, $\alpha$ is a constant chosen so that the righthand side of eq 24 becomes the maximum, and $\beta$ is a constant properly chosen in the range of $0<$ $\beta<1$. Then the desired expression for $[\eta]^{(\mathrm{L})}$ is given by the maximum of the the right-hand side of eq 24 with eq 27 and may be written in the form,

$$
[\eta]^{(\mathrm{L})}=\frac{N_{\mathrm{A}}}{M \eta_{0} g^{2} k_{\mathrm{B}} T}\left[\frac{\left\langle\mathbf{V}^{T} \cdot\left(\mathbf{D}_{\mathrm{p}}^{-1}+\mathbf{D}_{\mathrm{p}}^{\prime-1}\right) \cdot \mathbf{V}\right\rangle^{2}}{\left\langle\mathbf{V}^{T} \cdot\left(\mathbf{D}_{\mathrm{p}}^{-1}+\mathbf{D}_{\mathrm{p}}^{\prime-1}\right) \cdot \mathbf{D} \cdot\left(\mathbf{D}_{\mathrm{p}}^{-1}+\mathbf{D}_{\mathrm{p}}^{\prime-1}\right) \cdot \mathbf{V}\right\rangle}\right]
$$

As for the value of the constant $\beta$, it has been shown by Freire and Rey ${ }^{6}$ that in the case of Gaussian star chains $[\eta]^{(\mathrm{L})}$ given by eq 27 is insensitive to the value in the range of $0<\beta<1$ and therefore $\beta$ may be set equal to 0.5 . Following them, we have put $\beta=0.5$.

\section{RESULTS AND DISCUSSION}

We have carried out MC runs to generate sample configurations for the regular three-arm star and linear freely rotating chains with $n(=3 m)=60-300$ and $\theta=109^{\circ}, 165^{\circ}$, and $175^{\circ}$, with the parameters $\sigma$ and $T^{*}$ for the LJ potential being fixed to be 1 and 3.72, respectively. The freely rotating chain with $\theta=109^{\circ}$ corresponds to a flexible polymer and that with $\theta=165^{\circ}$ or $175^{\circ}$ to a semiflexible or stiff polymer. The value 3.72 of $T^{*}$ was previously ${ }^{13}$ determined so that $\left\langle S^{2}\right\rangle / n$ for the linear chain with $\Theta=109^{\circ}$ became a constant independent of $n$ for very large $n$, and therefore it corresponds to the $\Theta$ temperature, as mentioned in the previous section. For each chain, 5 MC runs have been carried out to obtain $10^{4}$ sample configurations, so that $5 \times 10^{4}$ sample configurations have been generated in total. We note that we have adopted $N=10^{4}$ in the present study of $[\eta]$, which is one order of magnitude smaller than $10^{5}$ adopted in the previous study of $\left\langle S^{2}\right\rangle,{ }^{13}$ since the computation time necessary for each sample configuration is much longer for $[\eta]$ than for $\left\langle S^{2}\right\rangle$.

On the basis of the sample configurations so gener- 
Table I. Results of Monte Carlo simulations for $\left\langle S^{2}\right\rangle / n$

\begin{tabular}{|c|c|c|c|c|}
\hline \multirow[b]{2}{*}{$n$} & \multicolumn{2}{|c|}{ three-arm star } & \multicolumn{2}{|c|}{ linear } \\
\hline & $\left\langle S^{2}\right\rangle / n$ (error \%) & $\begin{array}{l}\text { Acceptance } \\
\text { fraction }\end{array}$ & $\left\langle S^{2}\right\rangle / n$ (error \%) & $\begin{array}{l}\text { Acceptance } \\
\text { fraction }\end{array}$ \\
\hline \multicolumn{5}{|c|}{$\theta=109^{\circ}$} \\
\hline 60 & $0.296_{4}(0.5)$ & $59 / 200$ & $0.367_{2}(0.6)$ & $60 / 100$ \\
\hline 120 & $0.307_{2}(0.4)$ & $174 / 1000$ & $0.383_{2}(0.3)$ & $151 / 300$ \\
\hline 180 & $0.309_{9}(0.4)$ & $243 / 2000$ & $0.389_{6}(0.7)$ & $226 / 500$ \\
\hline 240 & $0.309_{0}(0.7)$ & $459 / 5000$ & $0.395_{1}(0.3)$ & $416 / 1000$ \\
\hline \multicolumn{5}{|c|}{$\theta=165^{\circ}$} \\
\hline 60 & $1.88_{6} \quad(0.1)$ & $199 / 200$ & $3.57_{1} \quad(0.1)$ & $99 / 100$ \\
\hline 120 & $3.02_{8} \quad(0.2)$ & $995 / 1000$ & $5.28_{5} \quad(0.2)$ & $299 / 300$ \\
\hline 180 & $3.81_{6} \quad(0.1)$ & $1979 / 2000$ & $6.26_{7} \quad(0.3)$ & $498 / 500$ \\
\hline 240 & $4.38_{0} \quad(0.1)$ & $4919 / 5000$ & $6.91_{0} \quad(0.4)$ & $993 / 1000$ \\
\hline \multicolumn{5}{|c|}{$\theta=175^{\circ}$} \\
\hline 60 & $2.29_{2} \quad(0.0)$ & $199 / 200$ & $4.93_{4} \quad(0.0)$ & $100 / 100$ \\
\hline 120 & $4.34_{2} \quad(0.0)$ & $999 / 1000$ & $9.29_{6} \quad(0.0)$ & $300 / 300$ \\
\hline 180 & $6.28_{9} \quad(0.0)$ & $1999 / 2000$ & 13.29 & $500 / 500$ \\
\hline 240 & $8.14_{1} \quad(0.0)$ & $4999 / 5000$ & $16.9_{7}$ & $999 / 1000$ \\
\hline 300 & $9.89_{8} \quad(0.1)$ & $4999 / 5000$ & $20.3_{4}$ & $999 / 1000$ \\
\hline
\end{tabular}

ated, we first examine the behavior of the MC values of $\left\langle S^{2}\right\rangle$ as a function of $n$ and compare them with the theoretical values for the ideal freely rotating chain without interactions between beads, which we call the ideal chain hereafter, and for the corresponding KP chain. Then we examine the behavior of $g_{S}$ as a function of the reduced contour length $\lambda L$, i.e., the total contour length $L$ of the corresponding KP chain divided by its the stiffness parameter $\lambda^{-1}$ having the dimension of length. Finally, we examine the behavior of $g_{\eta}$ as a function of $\lambda L$, which is the main purpose of the present study.

\section{Mean-Square Radius of Gyration}

In Table I are given the MC values of $\left\langle S^{2}\right\rangle / n$ for both the regular three-arm star and linear chains along with the values of the acceptance fraction, i.e., the mean number of changes in configuration in the $M_{\text {nom }}$ pivot steps divided by $M_{\text {nom }}$. Specifically, for example, for the regular three-arm star chain with $n=$ $60(m=20), 5$ independent MC runs have been repeated, in each of which $200 \times 10^{4}$ pivot steps have

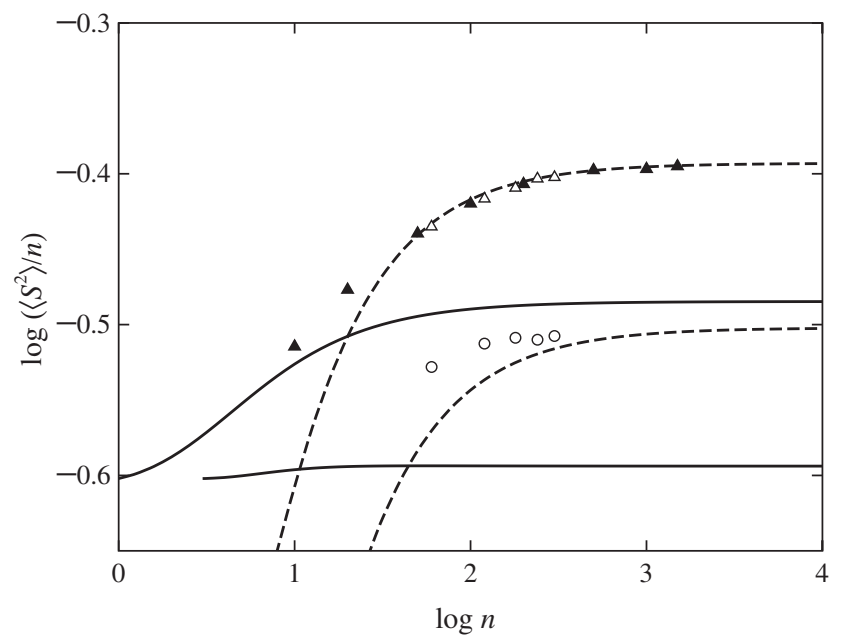

Figure 2. Double-logarithmic plots of $\left\langle S^{2}\right\rangle / n$ against $n$ for the regular three-arm star and linear chains, both with $\theta=109^{\circ}$ and $\sigma=1$ at $T^{*}=3.72$. The unfilled circles and triangles represent the present MC data for the regular three-arm star and the linear chains, respectively, and the filled triangles represent the previous MC data ${ }^{13}$ for the latter. The lower and upper solid line segments connect the theoretical values of the regular three-arm star and linear ideal freely rotating chains, respectively. The lower and upper dashed curve represent the theoretical values of the corresponding KP regular three-arm star and linear chains, respectively.

resulted in $59 \times 10^{4}$ changes in configuration. The values of $\left\langle S^{2}\right\rangle / n$ and its statistical error given in the second column for the star and the fourth for the linear are those of the mean and the standard deviation, respectively, of the results of the 5 independent MC runs.

Figure 2 shows double-logarithmic plots of $\left\langle S^{2}\right\rangle / n$ against $n$ with the present MC data given in Table I for the regular three-arm star $(\bigcirc)$ and linear $(\triangle)$ chains with $\theta=109^{\circ}$ along with the previous MC data for the linear chains $(\boldsymbol{\Delta}) .{ }^{13}$ Although the present number of sample configurations is smaller than the previous one, the unfilled and filled triangles seem to form a single-composite curve, indicating that the present data are so accurate as the previous ones.

In the figure, the lower and upper solid line segments connect the theoretical values for the regular three-arm star and linear ideal chains, respectively. The former values have been calculated from

$$
\begin{aligned}
\left\langle S^{2}\right\rangle= & \frac{7}{54} \frac{1-\cos \theta}{1+\cos \theta} n+\frac{1}{54} \frac{31+90 \cos \theta-13 \cos ^{2} \theta}{(1+\cos \theta)^{2}}+\frac{1}{18} \frac{19+49 \cos \theta+71 \cos ^{2} \theta+5 \cos ^{3} \theta}{(1+\cos \theta)^{3}} \frac{1}{n+1} \\
& -\frac{2(1+2 \cos \theta)}{(1+\cos \theta)^{3}} \frac{1-(-\cos \theta)^{n / 3+1}}{n+1}+\frac{1}{27} \frac{(1-\cos \theta)\left(37-16 \cos \theta+\cos ^{2} \theta\right)}{(1+\cos \theta)^{3}} \frac{1}{(n+1)^{2}} \\
& -\frac{2(2+\cos \theta)}{(1+\cos \theta)^{4}} \frac{1-(-\cos \theta)^{n / 3+1}}{(n+1)^{2}}+\frac{3}{(1+\cos \theta)^{4}} \frac{\left[1-(-\cos \theta)^{n / 3+1}\right]^{2}}{(n+1)^{2}} \quad \text { (regular three-arm star) }
\end{aligned}
$$


which is a special case of the theoretical expression obtained by Guenza et al. ${ }^{29}$ for regular $f$-arm star freely rotating chain, and the latter from

$$
\begin{aligned}
\left\langle S^{2}\right\rangle= & \frac{1}{6} \frac{1-\cos \theta}{1+\cos \theta} n+\frac{1}{6} \frac{1+6 \cos \theta-\cos ^{2} \theta}{(1+\cos \theta)^{2}}+\frac{1}{6} \frac{-1-7 \cos \theta+7 \cos ^{2} \theta+\cos ^{3} \theta}{(1+\cos \theta)^{3}} \frac{1}{n+1} \\
& -\frac{2 \cos ^{2} \theta}{(1+\cos \theta)^{4}} \frac{1-(-\cos \theta)^{n+1}}{(n+1)^{2}} \text { (linear) }
\end{aligned}
$$

It is seen for both the regular three-arm star and linear chains that the MC values are $c a .20 \%$ larger than the corresponding ideal-chain values in the limit of $n \rightarrow \infty$. This is due to the fact that the unperturbed $(\Theta)$ dimension of a polymer chain may be considerably enlarged by nonbonded interactions. ${ }^{30}$

In Figure 2 are also shown the theoretical values for the KP regular three-arm star and linear chains, the lower and upper dashed curves representing the respective values. The former values have been calculated from

$$
\left\langle S^{2}\right\rangle=\frac{7 L}{54 \lambda}-\frac{1}{3 \lambda^{2}}+\frac{1}{4 \lambda^{3} L}+\frac{1}{4 \lambda^{3}}\left(1-e^{-2 \lambda L / 3}\right)-\frac{3}{16 \lambda^{4} L^{2}}\left(1-e^{-4 \lambda L / 3}\right)
$$

(KP regular three-arm star)

which is a special case of the theoretical expression obtained by Mansfield and Stockmayer ${ }^{4}$ for a general KP star chain, and the latter from ${ }^{31}$

$$
\left\langle S^{2}\right\rangle=\frac{L}{6 \lambda}-\frac{1}{4 \lambda^{2}}+\frac{1}{4 \lambda^{3} L}-\frac{1}{8 \lambda^{4} L^{2}}\left(1-e^{-2 \lambda L}\right) \quad(\text { KP linear })
$$

In order to plot the KP theoretical values in the figure, we have converted $L$ to $n$ by the use of the relation

$$
\log n=\log (\lambda L)+\log \left(\lambda^{-1} n_{\mathrm{L}}\right)
$$

where $n_{\mathrm{L}}=n / L$ is the number of bonds per unit contour length, and used the values $3.0_{1}$ and $1.2_{4}$ of $\lambda^{-1}$ and $n_{\mathrm{L}}$, respectively, which values were previously ${ }^{13}$ determined in such a way that the theoretical values of the KP linear chain could most closely reproduce the previous MC values for the linear chain $(\mathbf{\Delta})$ with $n \gtrsim 50$.

Figures 3 and 4 show similar plots for the regular three-arm star and linear chains with $\theta=165^{\circ}$ and

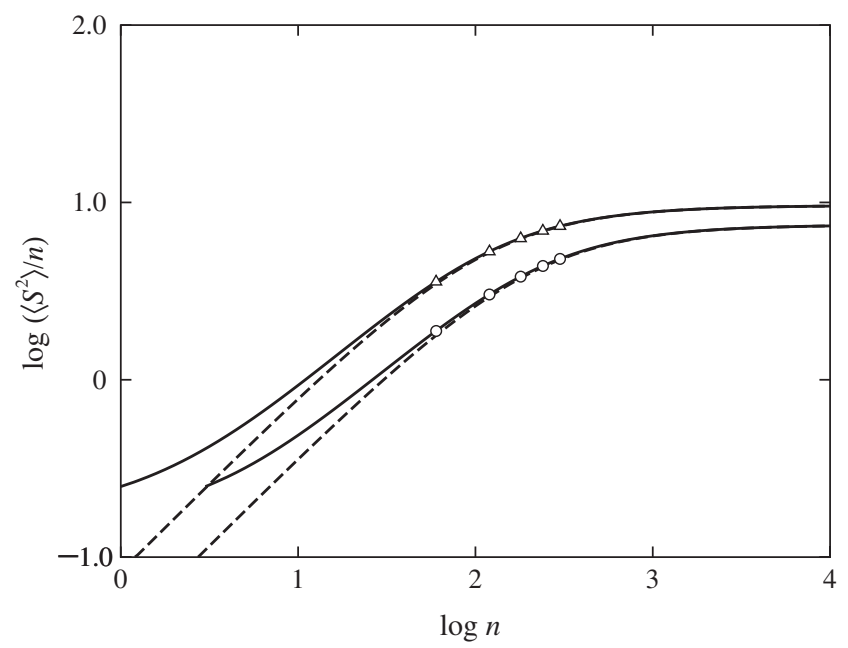

Figure 3. Double-logarithmic plots of $\left\langle S^{2}\right\rangle / n$ against $n$ for the regular three-arm star and linear chains, both with $\theta=165^{\circ}$ and $\sigma=1$ at $T^{*}=3.72$. All the symbols, line segments, and curves have the same meaning as those in Figure 2. $175^{\circ}$, respectively, which values of $\theta$ correspond to a semiflexible or stiff chain. All the symbols, line segments, and curves in the figures have the same meaning as those in Figure 2. It is seen that the MC values in each figure are almost identical with the corresponding ideal-chain values in contrast to the case of $\theta=109^{\circ}$ which corresponds to a flexible chain. This may be arising from the fact that effects of intramolecular interactions between beads become small with increasing chain stiffness if the total chain length is not very long. We therefore determine the values of the parameters $\lambda^{-1}$ and $n_{\mathrm{L}}$ of the corresponding KP chain in each case of $\theta$ from an analysis of the linear

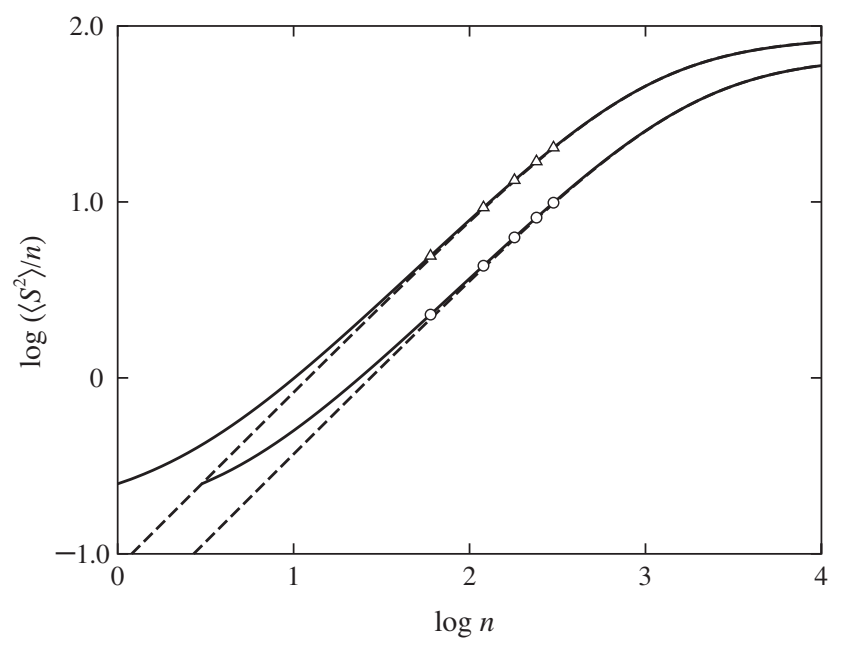

Figure 4. Double-logarithmic plots of $\left\langle S^{2}\right\rangle / n$ against $n$ for the regular three-arm star and linear chains, both with $\theta=175^{\circ}$ and $\sigma=1$ at $T^{*}=3.72$. All the symbols, line segments, and curves have the same meaning as those in Figure 2. 
Table II. Values of the KP model parameters

\begin{tabular}{cll}
\hline$\theta$ & \multicolumn{1}{c}{$\lambda^{-1}$} & $n_{\mathrm{L}}$ \\
\hline $109^{\circ}$ & $3.0^{\mathrm{a}}$ & $1.2_{4^{\mathrm{a}}}$ \\
$165^{\circ}$ & $5.7_{7} \times 10$ & $1.0_{0}$ \\
$175^{\circ}$ & $5.2_{6} \times 10^{2}$ & $1.0_{0}$ \\
\hline
\end{tabular}

${ }^{a}$ These values have been determined in the previous study. ${ }^{13}$

ideal-chain values with $n \gtrsim 10^{2}$. In each of Figures 3 and 4, the upper dashed curve represents the best-fit $\mathrm{KP}$ values calculated from eqs 34 and 35 with the parameter values given in Table II. It is seen that the theoretical values for the KP regular three-arm star (the lower dashed curve in each figure) calculated from eqs 33 and 35 with those parameter values may well reproduce the corresponding $\mathrm{MC}$ values. We note that the $\lambda^{-1}$ value so determined is $1.3 \%$ larger than the $\lambda^{-1}$ value calculated from the (ideal) relation $\lambda^{-1}=2 /(1+\cos \theta)$ in the case of $\theta=109^{\circ}$ and becomes identical with the latter value for $\theta \geq 165^{\circ}$.

Now we proceed to examine the behavior of $g_{S}$ as a function of $\lambda L$. Values of $g_{S}$ are plotted against $\log (\lambda L)$ in Figure 5. The three sets of the five open circles represent the $\mathrm{MC}$ values with the indicated values of $\theta$, calculated from the $\left\langle S^{2}\right\rangle$ values for the regular three-arm star and linear chains given in Table I with $n$ converted to $\lambda L$ by the use of eq 35 with the values of $\lambda^{-1}$ and $n_{\mathrm{L}}$ given in Table II. The solid line segments associated with each set of the MC data points connect the corresponding ideal-chain values calculated from eqs 31 and 32 with $n$ converted to $\lambda L$ in the above-mentioned manner. The dashed curve represents the KP theoretical values calculated from eq 33 and 34, with the upper and lower dotted horizontal lines representing the asymptotic values $7 / 9$ in the (ideal) random-coil limit and 4/9 in the rigidrod limit, respectively.

In the cases of $\theta=165^{\circ}$ and $175^{\circ}$ (semiflexible chain), the MC and ideal-chain values are almost identical with each other as a necessary consequence of the good agreement between the MC and idealchain values of $\left\langle S^{2}\right\rangle$ (see Figures 3 and 4). A remarkable point is that the two kinds of $g_{S}$ values for $\theta=109^{\circ}$ semiquantitatively agree with each other in spite of the rather large disagreement between the MC and ideal-chain values of $\left\langle S^{2}\right\rangle$ (see Figure 2). Both the MC and ideal-chain values for $\theta=109^{\circ}$ monotonically decrease with increasing $n$ and approach the asymptotic value 7/9 in the limit of $n \rightarrow \infty$, while the KP value monotonically increases.

\section{Intrinsic Viscosity}

It is convenient to present $\mathrm{MC}$ results for $[\eta]$ in terms of the dimensionless quantity $X_{\eta}$ defined by

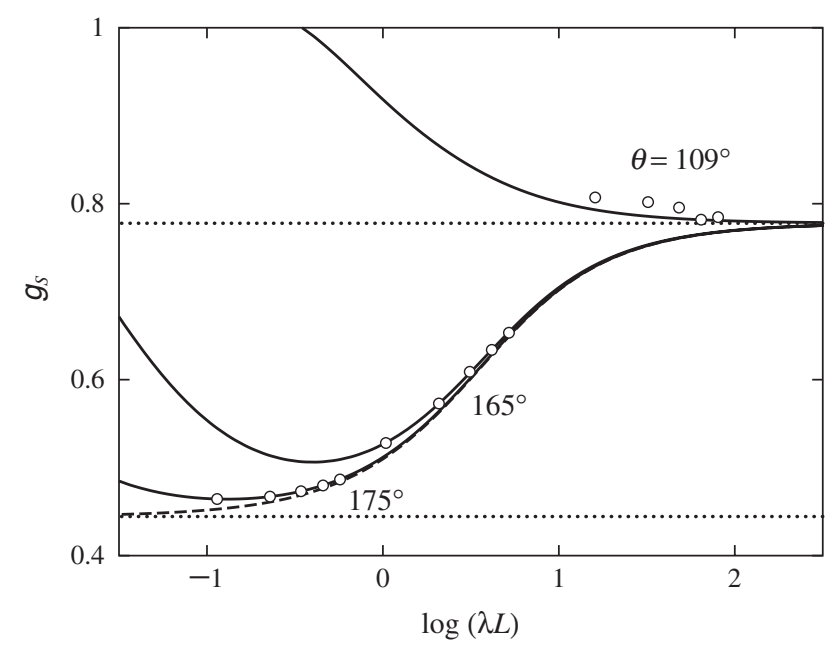

Figure 5. Plots of $g_{S}$ against $\log (\lambda L)$ for the regular three-arm star. The three sets of the five unfilled circles represent the MC values with the indicated values of $\theta$. The solid line segments associated with each set of the MC data points connect the corresponding theoretical values of the ideal freely rotating and the dashed curve represents the KP theoretical values. ${ }^{4}$ The upper and lower dotted horizontal lines represent the asymptotic value $7 / 9$ in the (ideal) random-coil limit and 4/9 in the rigid-rod limit, respectively.

$$
[\eta]=\frac{3 \pi N_{\mathrm{A}} n^{3 / 2}}{2 M} X_{\eta}
$$

As in the case of $[\eta]$, the superscript (KR), (U), and (L) are used to indicate that the $X_{\eta}$ values have been evaluated in the KR approximation, the Zimm rigid-body ensemble approximation, and the Fixman method, respectively. In Table III are given the values of $X_{\eta}^{(\mathrm{KR})}$, $X_{\eta}^{(\mathrm{U})}$, and $X_{\eta}^{(\mathrm{L})}$ along with their statistical errors, which are the mean and the standard deviation, respectively, of the results of the 5 independent MC runs, for both the regular three-arm star and linear chains.

Corresponding to the three kinds of $X_{\eta}$ (or $[\eta]$ ), we may consider the three kinds of $g_{\eta}$, i.e., $g_{\eta}^{(\mathrm{KR})}, g_{\eta}^{(\mathrm{Z})}$, and $g_{\eta}^{(\mathrm{F})}$ defined by

$$
\begin{aligned}
g_{\eta}^{(\mathrm{KR})} & =\frac{[\eta]^{(\mathrm{KR})}(\mathrm{star})}{[\eta]^{(\mathrm{KR})}(\text { linear })}, \\
g_{\eta}^{(\mathrm{Z})} & =\frac{[\eta]^{(\mathrm{U})}(\text { star })}{[\eta]^{(\mathrm{U})}(\text { linear })}, \\
g_{\eta}^{(\mathrm{F})} & =\frac{[\eta]^{(\mathrm{L})}(\text { star })}{[\eta]^{(\mathrm{L})}(\text { linear })}
\end{aligned}
$$

We note that $g_{\eta}^{(\mathrm{Z})}$ is not an upper bound to $g_{\eta}$, nor is $g_{\eta}^{(\mathrm{F})}$ a lower bound. Figure 6 shows plots of $g_{\eta}^{(\mathrm{KR})}$ $(\bigcirc), g_{\eta}^{(\mathrm{Z})}(\triangle)$, and $g_{\eta}^{(\mathrm{F})}(\nabla)$ against $\log (\lambda L)$. Three sets of the data points represent the MC values with the indicated values of $\theta$, calculated from eqs 36 and 37 with the three kinds of $X_{\eta}$ values given in Table III and with $n$ converted to $\lambda L$ in the above-mentioned manner. It is interesting to see that the $g_{\eta}^{(\mathrm{Z})}$ and $g_{\eta}^{(\mathrm{F})}$ 
$[\eta]$ of Semiflexible Three-Arm Star Polymers

Table III. Values of $X_{\eta}^{(\mathrm{KR})}, X_{\eta}^{(\mathrm{U})}$, and $X_{\eta}^{(\mathrm{L})}$

\begin{tabular}{|c|c|c|c|c|c|c|}
\hline \multirow{2}{*}{$n$} & \multicolumn{3}{|c|}{ three-arm star } & \multicolumn{3}{|c|}{ linear } \\
\hline & $X_{\eta}^{(\mathrm{KR})}($ error \%) & $X_{\eta}^{(\mathrm{U})}($ error $\%)$ & $X_{\eta}^{(\mathrm{L})}($ error \%) & $X_{\eta}^{(\mathrm{KR})}($ error \%) & $X_{\eta}^{(\mathrm{U})}($ error \%) & $X_{\eta}^{(\mathrm{L})}($ error \%) \\
\hline \multicolumn{7}{|c|}{$\theta=109^{\circ}$} \\
\hline 60 & $0.304_{1}(0.3)$ & $0.272_{4}(0.5)$ & $0.250_{6}(0.4)$ & $0.333_{3}(0.3)$ & $0.306_{9}(0.3)$ & $0.283_{0}(0.3)$ \\
\hline 120 & $0.321_{2}(0.4)$ & $0.284_{4}(0.4)$ & $0.255_{9}(0.4)$ & $0.352_{8}(0.4)$ & $0.321_{1}(0.4)$ & $0.289_{8}(0.5)$ \\
\hline 180 & $0.329_{0}(0.4)$ & $0.288_{3}(0.4)$ & $0.256_{6}(0.6)$ & $0.363_{2}(0.5)$ & $0.329_{3}(0.6)$ & $0.293_{1}(0.6)$ \\
\hline 240 & $0.332_{9}(0.5)$ & $0.290_{3}(0.6)$ & $0.255_{4}(0.8)$ & $0.369_{7}(0.2)$ & $0.335_{0}(0.3)$ & $0.295_{1}(0.3)$ \\
\hline 300 & $0.336_{0}(0.7)$ & $0.293_{3}(0.8)$ & $0.255_{5}(0.9)$ & $0.374_{3}(0.5)$ & $0.338_{7}(0.5)$ & $0.295_{7}(0.6)$ \\
\hline \multicolumn{7}{|c|}{$\theta=165^{\circ}$} \\
\hline 60 & $1.76_{3} \quad(0.3)$ & $1.77_{8} \quad(0.2)$ & $1.72_{6} \quad(0.3)$ & $2.91_{1} \quad(0.5)$ & $2.96_{1} \quad(0.5)$ & $2.88_{0} \quad(0.5)$ \\
\hline 120 & $3.21_{7} \quad(0.2)$ & $3.23_{7} \quad(0.3)$ & $3.11_{9} \quad(0.2)$ & $5.02_{2} \quad(0.3)$ & $5.13_{5} \quad(0.3)$ & $4.95_{0} \quad(0.3)$ \\
\hline 180 & $4.45_{5} \quad(0.3)$ & $4.47_{8} \quad(0.2)$ & $4.28_{7} \quad(0.3)$ & $6.63_{4} \quad(0.3)$ & $6.76_{5} \quad(0.4)$ & $6.49_{1} \quad(0.4)$ \\
\hline 240 & $5.51_{0} \quad(0.4)$ & $5.52_{1} \quad(0.2)$ & $5.26_{4} \quad(0.4)$ & $7.94_{6} \quad(0.7)$ & $8.07_{1} \quad(0.7)$ & $\begin{array}{ll}7.72_{1} \quad(0.7)\end{array}$ \\
\hline 300 & $6.41_{3} \quad(0.3)$ & $6.37_{9} \quad(0.3)$ & $6.08_{8} \quad(0.3)$ & $9.06_{2} \quad(0.5)$ & $9.16_{1} \quad(0.5)$ & $8.74_{5} \quad(0.5)$ \\
\hline \multicolumn{7}{|c|}{$\theta=175^{\circ}$} \\
\hline 60 & $2.12_{9} \quad(0.1)$ & $2.12_{7} \quad(0.1)$ & $2.08_{7} \quad(0.1)$ & $3.98_{8} \quad(0.6)$ & $4.02_{0} \quad(0.5)$ & $3.89_{9} \quad(0.5)$ \\
\hline 120 & $4.57_{2} \quad(0.2)$ & $4.59_{5} \quad(0.1)$ & $4.46_{8} \quad(0.2)$ & $8.70_{8} \quad(0.3)$ & $8.83_{2} \quad(0.4)$ & $8.51_{6} \quad(0.4)$ \\
\hline 180 & $7.26_{3} \quad(0.2)$ & $\begin{array}{ll}7.32_{7} & (0.2)\end{array}$ & $7.09_{2} \quad(0.2)$ & $13.8_{0} \quad(0.4)$ & $14.0_{6}$ & $13.5_{2} \quad(0.3)$ \\
\hline 240 & $10.0_{8} \quad(0.2)$ & $10.2_{3}$ & $9.84_{8} \quad(0.3)$ & $19.0_{6}$ & $19.4_{9}$ & $18.7_{0}$ \\
\hline 300 & $13.0_{1} \quad(0.2)$ & $13.2_{4}$ & $12.7_{1} \quad(0.3)$ & $24.2_{8}$ & $24.9_{3}$ & $23.8_{7}$ \\
\hline
\end{tabular}

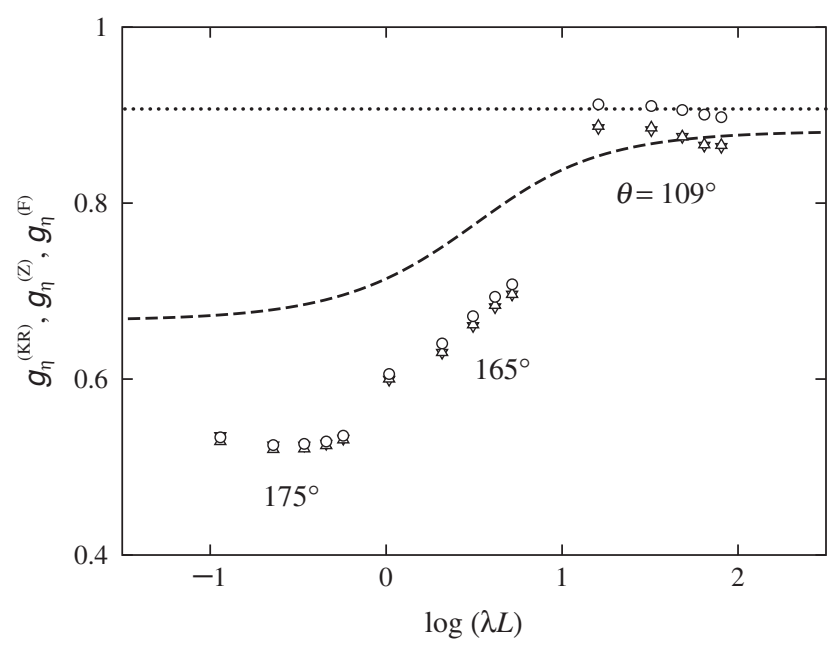

Figure 6. Plots of $g_{\eta}^{(\mathrm{KR})}, g_{\eta}^{(\mathrm{Z})}$, and $g_{\eta}^{(\mathrm{F})}$ against $\log (\lambda L)$. Three sets of the five unfilled circles, the five unfilled triangles, and the five unfilled inverted triangles represent the MC data of $g_{\eta}^{(\mathrm{KR})}, g_{\eta}^{(\mathrm{Z})}$, and $g_{\eta}^{(\mathrm{F})}$, respectively, with the indicated values of $\theta$. The dotted horizontal line represents the Zimm-Kilb ${ }^{2}$ value $0.90_{7}$ for the Gaussian chain and the dashed curve the values of $g_{S}{ }^{1 / 2}$ for the KP chain.

values almost completely agree with each other irrespective of the value of $\lambda L$, i.e., chain stiffness $(\theta)$ and total chain length $(n)$, although the $X_{\eta}^{(\mathrm{U})}$ and $X_{\eta}^{(\mathrm{L})}$ values themselves do not. As for $g_{\eta}^{(\mathrm{KR})}$, its value agrees well with the $g_{\eta}^{(\mathrm{Z})}$ and $g_{\eta}^{(\mathrm{F})}$ values for very small $\lambda L$, i.e., for $\theta=175^{\circ}$ with small $n$, but deviates upward from those with increasing $\lambda L$. In the case of $\theta=$ $109^{\circ}$ (flexible chain), $g_{\eta}^{(\mathrm{KR})}$ values are $c a .5 \%$ larger than the corresponding $g_{\eta}^{(\mathrm{Z})}$ and $g_{\eta}^{(\mathrm{F})}$ values.

Available theoretical asymptotic values of $g_{\eta}$ in the (ideal) random-coil limit $(n \rightarrow \infty$ or $\lambda L \rightarrow \infty)$ are $0.90_{7}$ obtained by Zimm and $\mathrm{Kilb}^{2}$ for the (dynamic) Gaussian spring-bead model with the preaveraged Oseen tensor and 0.90 obtained by Irurzun ${ }^{32}$ for the Gaussian chain in the KR approximation. In Figure 6, the dotted horizontal line represents the Zimm-Kilb value $0.90_{7}$. Naturally, the $g_{\eta}^{(\mathrm{KR})}$ values for $\theta=109^{\circ}$ agree well with the Zimm-Kilb value and also with the Irurzun one. If those MC values were appreciably different from the theoretical asymptotic values, the sample configurations of the regular three-arm star might not have been properly generated.

The approximate relation $g_{\eta} \simeq g_{S}{ }^{1 / 2}$ proposed by Zimm and $\mathrm{Kilb}^{2}$ for Gaussian branched chains has been often used in analyses of experimental data. The dashed curve in Figure 6 represents the values of $g_{S}{ }^{1 / 2}$ for the KP regular three-arm star chain calculated from eqs 33 and 34. It is seen that the approximate relation overestimates $g_{\eta}$ for semiflexible or stiff polymers.

Finally, we consider upper and lower bounds to $g_{\eta}$. From the inequality $[\eta]^{(\mathrm{L})} \leq[\eta] \leq[\eta]^{(\mathrm{U})}$ that holds for both the regular three-arm star and linear chains, we have the following two inequalities,

$$
\begin{aligned}
& g_{\eta} \leq g_{\eta}^{(\mathrm{U})}=\frac{[\eta]^{(\mathrm{U})}(\text { star })}{[\eta]^{(\mathrm{L})}(\text { linear })}, \\
& g_{\eta} \geq g_{\eta}^{(\mathrm{L})}=\frac{[\eta]^{(\mathrm{L})}(\text { star })}{[\eta]^{(\mathrm{U})}(\text { linear })}
\end{aligned}
$$

Figure 7 shows the plots of $g_{\eta}^{(\mathrm{KR})}(\bigcirc)$ against $\log (\lambda L)$ reproduced from Figure 6, where the upper and lower ends of each vertical error bar represent the $g_{\eta}^{(\mathrm{U})}$ and $g_{\eta}^{(\mathrm{L})}$ values, respectively, calculated from eqs 36 and 


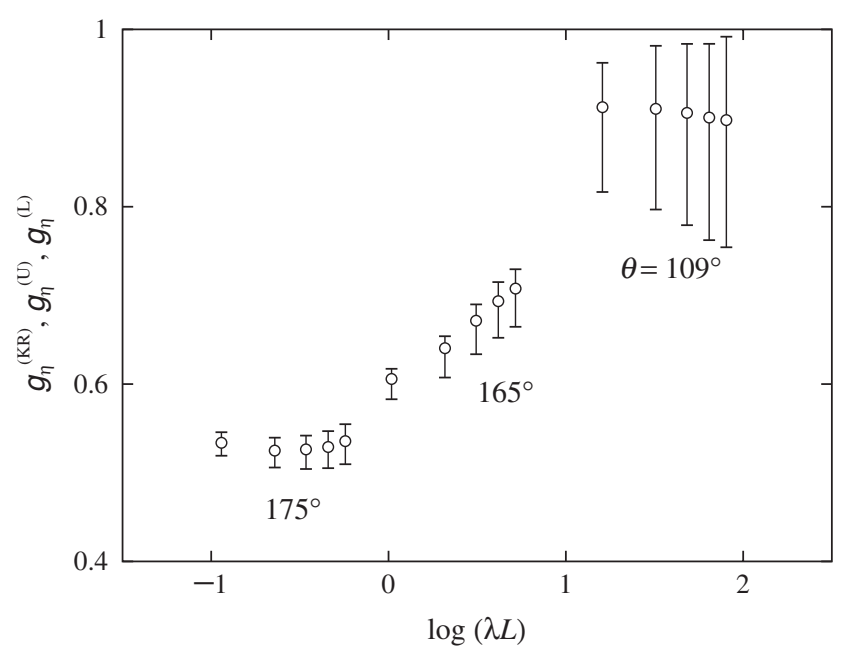

Figure 7. Plots of $g_{\eta}^{(\mathrm{KR})}, g_{\eta}^{(\mathrm{U})}$, and $g_{\eta}^{(\mathrm{L})}$ against $\log (\lambda L)$. The unfilled circles have the same meaning as those in Figure 6. The upper and lower ends of each vertical error bar represent the MC values of $g_{\eta}^{(\mathrm{U})}$ and $g_{\eta}^{(\mathrm{L})}$, respectively (see the text).

38 with the values of $X_{\eta}^{(\mathrm{U})}$ and $X_{\eta}^{(\mathrm{L})}$ given in Table III and with $n$ converted to $\lambda L$ in the above-mentioned manner. The difference between the upper and lower bounds increases with increasing $\lambda L$. Although the differences are unexpectedly large for flexible chains $\left(\theta=109^{\circ}\right)$, they are sufficiently small for semiflexible or stiff chains $\left(\theta=165^{\circ}, 175^{\circ}\right)$ to let us conclude that $g_{\eta}^{(\mathrm{KR})}$ may give a good approximate value for the latter chains.

\section{CONCLUDING REMARKS}

We have examined the behavior of the ratio $g_{\eta}$ of the intrinsic viscosity $[\eta]$ for the regular three-arm star chain to that for the linear one, both in the $\Theta$ state and having the same total chain length, by MC simulation of freely rotating chains with the LJ 6-12 potentials between beads in a cutoff version. On the basis of the values of the upper bound $[\eta]^{(\mathrm{U})}$ and lower one $[\eta]^{(\mathrm{L})}$ to $[\eta]$ evaluated by the Zimm rigid-body approximation and by the Fixman method, respectively, the values of the upper bound $g_{\eta}^{(\mathrm{U})}$ and lower one $g_{\eta}^{(\mathrm{L})}$ to $g_{\eta}$ have been estimated. It has then been found that the KR value $g_{\eta}^{(\mathrm{KR})}$ of $g_{\eta}$ lies between the two bounds irrespective of the number $n$ of bonds and the bond angle $\theta$ (or the reduced contour length $\lambda L$ ). Although the differences between the two bounds are unexpectedly large for flexible chains $\left(\theta=109^{\circ}\right)$, they are sufficiently small for semiflexible or stiff chains $(\theta=$ $165^{\circ}, 175^{\circ}$ ) to let us conclude that $g_{\eta}^{(\mathrm{KR})}$ may give a good approximate value for the latter chains. We therefore proceed to make a theoretical study of $g_{\eta}$ for the unperturbed KP chain in the KR approximation and to construct an interpolation formula useful for an analysis of experimental data.

\section{REFERENCES AND NOTES}

1. B. H. Zimm and W. H. Stockmayer, J. Chem. Phys., 17, 1301 (1949).

2. B. H. Zimm and R. W. Kilb, J. Polym. Sci., 37, 19 (1959).

3. H. Yamakawa, in "Modern Theory of Polymer Solutions," Harper \& Row, New York, 1971. (URL: http://www.molsci. polym.kyoto-u.ac.jp/archives/redbook.pdf)

4. M. L. Mansfield and W. H. Stockmayer, Macromolecules, 13, 1713 (1980).

5. B. H. Zimm, Macromolecules, 17, 795 (1984).

6. J. J. Freire and A. Rey, Comput. Phys. Commum., 61, 297 (1990).

7. J. F. Douglas, J. Roovers, and K. F. Freed, Macromolecules, 23, 4168 (1990).

8. Y. Nakamura, Kobunshi Ronbunshu, 57, 530 (2000).

9. S. H. Goodson and B. M. Novak, Macromolecules, 34, 3849 (2001).

10. O. Kratky and G. Porod, Recl. Trav. Chim. Pays-Bas., 68, 1106 (1949).

11. H. Yamakawa, in "Helical Wormlike Chains in Polymer Solutions," Springer, Berlin, 1997.

12. B. H. Zimm, Macromolecules, 13, 592 (1980).

13. H. Yamakawa and T. Yoshizaki, J. Chem. Phys., 118, 2911 (2003).

14. H. Yamakawa and T. Yoshizaki, J. Chem. Phys., 119, 1257 (2003).

15. J. P. Hansen and I. R. McDonald, in "Theory of Simple Liquids," 3rd ed., Academic, London, 2006.

16. N. Metropolis, A. W. Rosenbluth, M. N. Rosenbluth, A. H. Teller, and E. Teller, J. Chem. Phys., 21, 1087 (1953).

17. M. Lal, Mol. Phys., 17, 57 (1969).

18. S. D. Stellman and P. J. Gans, Macromolecules, 5, 516 (1972).

19. N. Madras and A. D. Sokal, J. Stat. Phys., 50, 109 (1988).

20. J. G. Kirkwood and J. Riseman, J. Chem. Phys., 16, 565 (1948).

21. M. Fixman, J. Chem. Phys., 78, 1588 (1983).

22. M. Fixman, J. Chem. Phys., 78, 1594 (1983).

23. M. Matsumoto and T. Nishimura, ACM Trans. Model. Comput. Simul., 8, 3 (1998). (URL: http://www.math. keio.ac.jp/matsumoto/emt.html)

24. J. Rotne and S. Prager, J. Chem. Phys., 50, 4831 (1969).

25. H. Yamakawa, J. Chem. Phys., 53, 436 (1970).

26. R. E. DeWames, W. F. Hall, and M. C. Shen, J. Chem. Phys., 46, 2782 (1967).

27. R. Zwanzig, J. Kiefer, and G. H. Weiss, Proc. Natl. Acad. Sci. USA, 60, 381 (1968).

28. G. Wilemski and G. Tanaka, Macromolecules, 14, 1531 (1981).

29. M. Guenza, M. Mormino, and P. Perico, Macromolecules, 24, 6168 (1991).

30. H. Yamakawa and T. Yoshizaki, J. Chem. Phys., 121, 3295 (2004).

31. H. Benoit and P. Doty, J. Phys. Chem., 57, 958 (1953).

32. I. M. Irurzun, J. Polym. Sci., Part B: Polym. Phys., 37, 563 (1997). 